\title{
Image Quality Assessment using Image Details in Frequency Domain
}

\author{
MUHAMMAD IBRAR-UL-HAQUE*, MUHAMMAD TAHIR QADRI**, AND NAJEEB SIDDIQUI** \\ RECEIVED ON 19.12.2015 ACCEPTED ON 16.08.2016
}

\begin{abstract}
This research proposes a RR (Reduced Reference) DIQAM (Detailed Image Quality Assessment Meter) for DCT (Discrete Cosine Transform) based compressed images. DCT technique divides image in sub blocks to achieve image compression.Therefore, it degrades the IQ (Image Quality) by introducing the distortions called blockiness and blurriness in the compressed image.In the telecommunication systems scenario, the systems available bandwidth is limited. The proposed IQ assessment technique requires fewer image details parameters called RR parameters at the receiver, rather than the complete reference image. This paper suggests a method for receiving end to estimate the objective quality of the received image in frequency domain. The proposed IQ meter starts by taking the image through edge detection method, then converting it into frequency domain by Fourier transform and estimating the image details. The image details calculations include the vertical and horizontal ac harmonics as well as all other ac coefficients present in the image. It has been shown in the presented work that using dc coefficients with the other ac coefficients further improves the quality assessment.The calculated strength of coded image details at receiver is compared with the received $R R$ parameter for the estimation of distortions, blockiness and blurriness. The accuracy of the designed RR DIQAM algorithm is proved by correlating the estimated objective values of the distortions with the LIVE image database2 subjective DMOS values of blockiness and blurriness. The results obtained by the proposed technique are well matched with the LIVE database values and provide $94-96 \%$ correlation.
\end{abstract}

Key Words: Image Processing, Image Quality Assessment, DC Coefficients, AC Coefficients, Blockiness, Blurriness, Reduce Reference.

\section{INTRODUCTION}

M

easurement of picture quality is primarily important for many image processing applications. The existing IQ estimation methods can be divided into subjective [1] and objective methods [2]. The purpose of quality evaluation algorithm is to automatically estimate the objective image or video quality in precise agreement with subjective human visual quality judgment. The objective IQ meters are divided into the FR (Full Reference) mode, RR mode and NR (No Reference) mode [2,3]. The IQ degrades due to the

Corresponding Author: (E-Mail: mihaque@ssuet.edu.pk)

* Department of Electrical Engineering, Sir Syed University of Engineering \& Technology, Karachi.

** Department of Electronic Engineering, Sir Syed University of Engineering \& Technology, Karachi.

Mehran University Research Journal of Engineering \& Technology, Volume 36, No. 4, October, 2017 [p-ISSN: 0254-7821, e-ISSN: 2413-7219] 
different compression techniques. The DCT based JPEG [4] and WT (Wavelet Transform) based PEG2000 are different standards of image compression [5]. The DCT based procedure is lossy, which introduces different distortions or artefacts due to the block based compression [6,7]. These artefacts are blockiness and blurriness. At block boundaries, discontinuities come from the blockiness due to the process of quantization and blurriness reduces the image sharpness [4].

Number of researchers have adopted different methodologies and contributed substantial study in the area of picture quality assessment, and progress is made in their particular domains. The algorithm's objective IQ assessments should be closely matched or highly correlated with subjective human judgments, despite of the kind of artefact corrupting the image. Usual FR IQ assessment method of PSNR (Peak Signal to Noise Ratio) and MSE (Mean Square Error) [8], between a compressed image and related reference are not in conformity with supposed quality measurements. Lei et. al. [9] have given FR mode FSIM (Feature Similarity) index reference IQ assessment method, established on the information that HVS (Human Visual System) uses mainly according to the image low level features. The primary element of FSIM is the PC (Phase Congruency), a dimensionless measure of the local structure. The image GM (Gradient Magnitude) is used as the secondary element for the contrast information, in FSIM. A frequency DM (Distortion Measure), and additive NQM (Noise Quality Measure) [10]. Total of 779 distorted images were evaluated by about 24 human subjects and subjective statistical evaluation in [11]. Several FR, IQ assessment algorithms performances are evaluated by IQ data obtained from about 25,000 human quality judgments. An IQ assessment technique using RR method is proposed in [12]. Distorted IQ is measured by comparing normalized histogram of both the distorted and reference image.
In this work we present the results of RR objective DIQAM method, using image details in frequency domain. The blockiness and blurriness distortions are measured as quality metrics. The evaluation of the performance of DIQAM algorithms is tested with LIVE image database2 [13] based on human IQ evaluation. The database contains the subjective scores for 233 blocky and 174 blurred images.

This paper is structured as follows: Section 2 gives the general system model for detailed IQ assessment. Section 3 represents the results of the algorithm, which are discussed in Section 2.1, 2.2 and 2.3. We wrap up our paper in Section IV.

\section{GENERAL SYSTEM MODEL FOR IMAGE QUALITY ASSESSMENT}

The system model proposes a DIQAM using image details in frequency domain in reduced or partial reference mode. In this technique instead of full image, only few of the reference parameters called RR parameters are required at receiver end for the judgment of DCT based coded IQ.DCT compression reduces the size of image, but due to block based technique, the blockiness and blurriness distortions are also introduced in the image.IQ is measured by calculating these two distortions.

To display the blockiness and blurriness artefacts in compressed images, the example image in Fig. 1, carnivaldolls.bmp is taken from LIVE image database 2 [13]. Fig. 1(a) shows the actual reference image, while its blocky and blurred versions are given in Fig. 1(b-c).

The proposed general DIQAM quality meter model is represented in Fig. 2. The process begins by reading the LIVE image in RGB format and converts it to grayscale. Only luminance information of the image is used for estimating the maximum or peak values of harmonics. At

Mehran University Research Journal of Engineering \& Technology, Volume 36, No. 4, October, 2017 [p-ISSN: 0254-7821, e-ISSN: 2413-7219] 
the block boundaries, discontinuities or abrupt changes (or luminance change in pixels) come from the blockiness or blurriness. Then the Sobel edge detection process is applied on the compressed image. It filters out the useless information by identifying the pixels discontinuities and the points in image at which the brightness changes sharply. After this the image is divided in 32x32 pixels blocks and then frequency domain transformation is performed for the image.

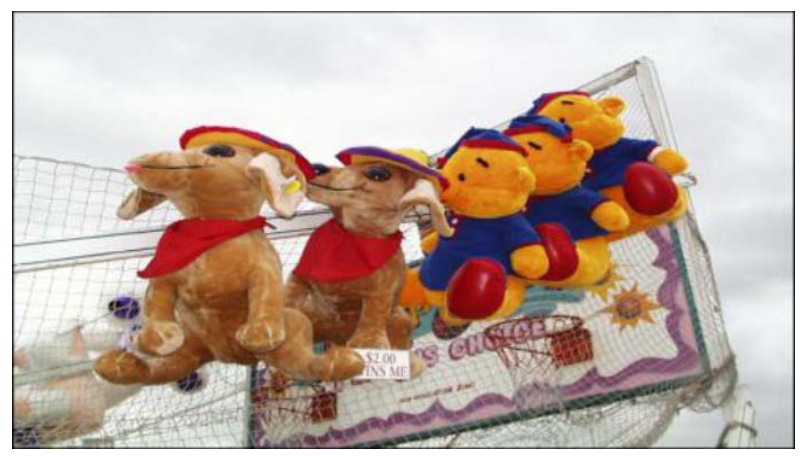

(a)REFERENCE IMAGE

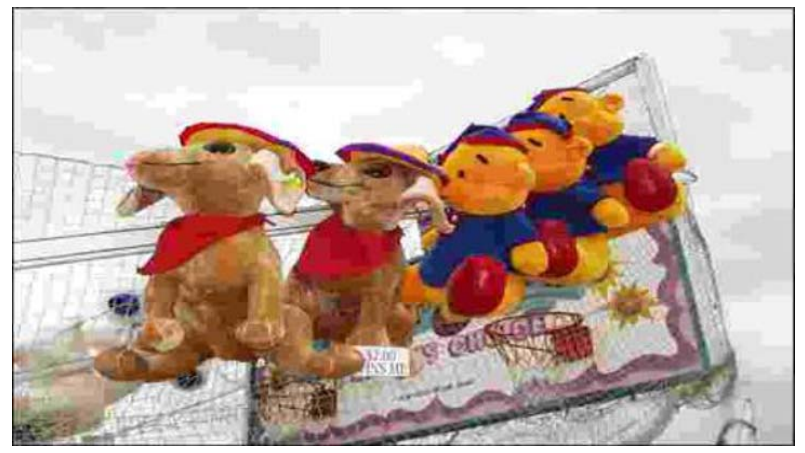

(b)BLOCKY IMAGE

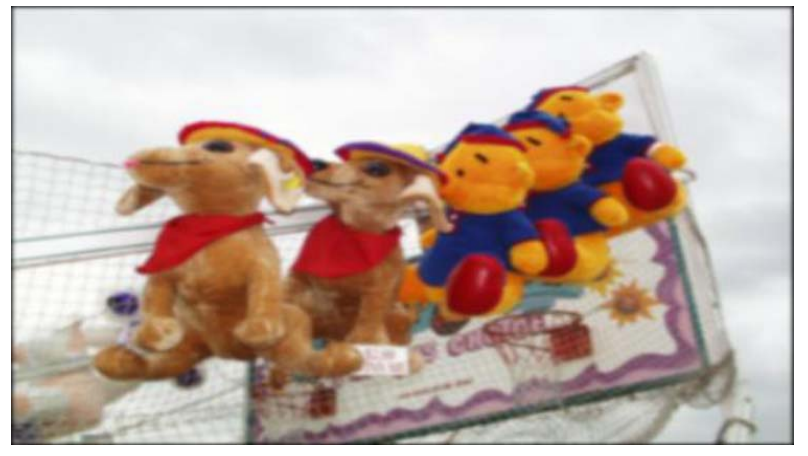

(c)BLURRY IMAGE

FIG. 1. CARNIVALDOLLS.BMP IMAGE [13]
The idea of blockiness is understood by taking the ideal 32x32 pixels blocky image of Fig. 3(a), has repetitive blocks in columns and rows. The harmonics are shown in its transformed version in Fig. 3(b). By taking FFT the horizontal, $\mathrm{H}$ and vertical, $\mathrm{V}$ harmonics are easily identified. The 32x32 pixels block size is chosen here, for the reason that it is multiple of 8x8 pixels fixed standard DCT block size. If 16x16 pixels block size is selected, then there are only two blockiness harmonics and are not sufficiently spaced out from each other. Therefore we picked 32x32 pixels size of the block in which there are $4^{\text {th }}$, $8^{\text {th }}$ and $12^{\text {th }}$, three harmonics (or coefficients) and are easily distinguishable. Because they are reasonably apart from each other and we are able to get more accurate results. Finally the different IQ assessment techniques are applied on of the transformed image.

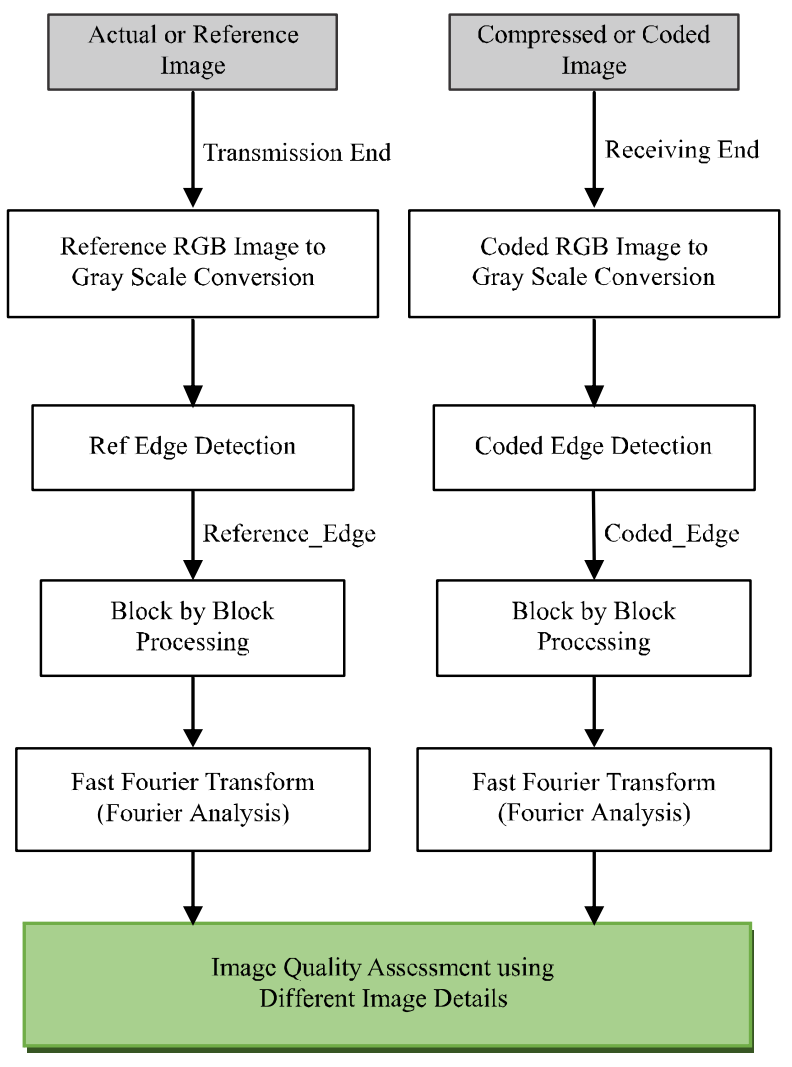

FIG. 2. GENERAL SYSTEM MODEL FOR DETAILED IMAGE QUALITY ASSESSMENT IN FREQUENCY DOMAIN

Mehran University Research Journal of Engineering \& Technology, Volume 36, No. 4, October, 2017 [p-ISSN: 0254-7821, e-ISSN: 2413-7219] 


\subsection{Image Quality Assessment using Harmonic Analysis in Frequency Domain}

Due to the fixed block size DCT based compression; harmonics are introduced in the image. These harmonics are repetitive in rows and columns. Therefore, the IQ assessment can be done by calculating the harmonics in horizontal and vertical directions. Fig. 4 illustrates the System model of IQAssessment in RR mode using harmonic analysis. From the general model of Fig. 2, after obtaining the transformed image by FFT block, the harmonics strength is calculated.

The harmonics strength ( $\left.\mathrm{HV}_{\text {strength_ref }}\right)$ of reference image at transmission end, also called RR parameter is computed by Equation (1) as [14]:

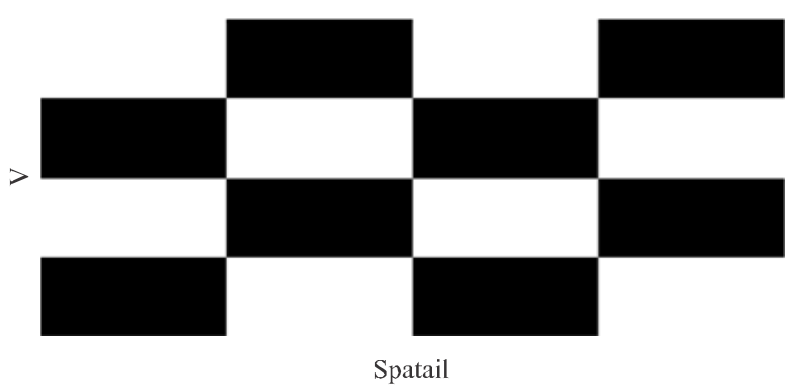

(a). REFERENCE IDEAL $32 X 32$ PIXEL BLOCKY IMAGE IN SPATIAL DOMAIN

Strength of Harmonics in Blocky blocks

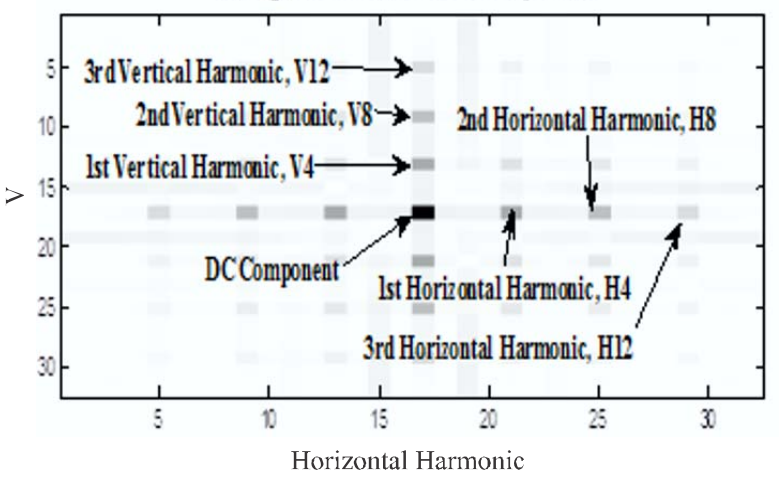

(b). FFT OF THE IDEAL $32 X 32$ PIXEL BLOCKY IMAGE SHOWING HARMONICS

FIG. 3. IDEAL BLOCKY IMAGE AND ITS FOURIER TRANSFORM [14]

$$
\mathrm{HV}_{\text {strength_ref }}=\frac{(\mathrm{V} 4+\mathrm{V} 8+\mathrm{V} 12) \mathrm{ref}}{\sum_{\mathrm{i}=1}^{16} \mathrm{~V}_{\mathrm{i}}}+\frac{(\mathrm{H} 4+\mathrm{H} 8+\mathrm{H} 12) \mathrm{ref}}{\sum_{\mathrm{i}=1}^{16} \mathrm{H}_{\mathrm{i}}}
$$

Where, $\mathrm{H}_{4}, \mathrm{H}_{8}, \mathrm{H}_{12}$ are horizontal and are $\mathrm{V}_{4}, \mathrm{~V}_{8}, \mathrm{~V}_{12}$ are vertical harmonics. Similarly, the harmonics strength $\left(\mathrm{HV}_{\text {strength_cod }}\right)$ for coded image at receiving end, is computed using Equation (2) as [14]:

$\mathrm{HV}_{\text {strength_cod }}=\frac{(\mathrm{V} 4+\mathrm{V} 8+\mathrm{V} 12) \operatorname{cod}}{\sum_{\mathrm{i}=1}^{16} \mathrm{~V}_{\mathrm{i}}}+\frac{(\mathrm{H} 4+\mathrm{H} 8+\mathrm{H} 12) \mathrm{cod}}{\sum_{\mathrm{i}=1}^{16} \mathrm{H}_{\mathrm{i}}}$

IQ metric can be defined by:

IQ = Blockiness Index + Blurriness Index

The blockiness and blurriness are obtained by different RR parameters, presented in Figs. 4-5,7 in the next sections.

The amplitude strength of these harmonics is increased (where blockiness occurs) or decreased (where blurriness occurs) in coded image. This is identified at the receiver, through the comparison of these harmonics strength obtained from coded and reference image. The ratio $\mathrm{HV}_{\text {strength_cod }}$ is compared with the single RR ratio parameter $\mathrm{HV}_{\text {strength_ref }}$ of reference image.

For model of Fig. 4, RR Blockiness is obtained by equation4, when $\mathrm{HV}_{\text {strength_cod }}>\mathrm{HV}_{\text {strength_ref, }}$

Blockiness $=\mathrm{HV}_{\text {strength_cod }}-\mathrm{HV}_{\text {strength_ref }}$

and Blurriness is found when $\mathrm{HV}_{\text {strength_cod }}<\mathrm{HV}_{\text {strength_ref }}$ by Equation (5)

Blurriness $=\left(\mathrm{HV}_{\text {strength_ref }}-\mathrm{HV}_{\text {strength_cod }}\right)$

By the use of Equation (3), total summation of these values of all blocks will produce the actual combined artefacts estimated objective blockiness/blurriness value, called IQ metric. 


\subsection{Image Quality Assessment using other ac Coefficients in Frequency Domain}

The system model of IQ assessment in reduced reference mode using other ac coefficients is represented in Fig. 5.

The image details are also present in remaining ac coefficients other than the horizontal and vertical harmonics in the image. Fig. 6 shows, other ac coefficients present in transformed image between horizontal and vertical harmonics of the 32x32 pixels ideal reference blocky image of Fig. 3(a).

The value of these remaining ac coefficients can also be used for IQ estimation. In the transformed image obtained

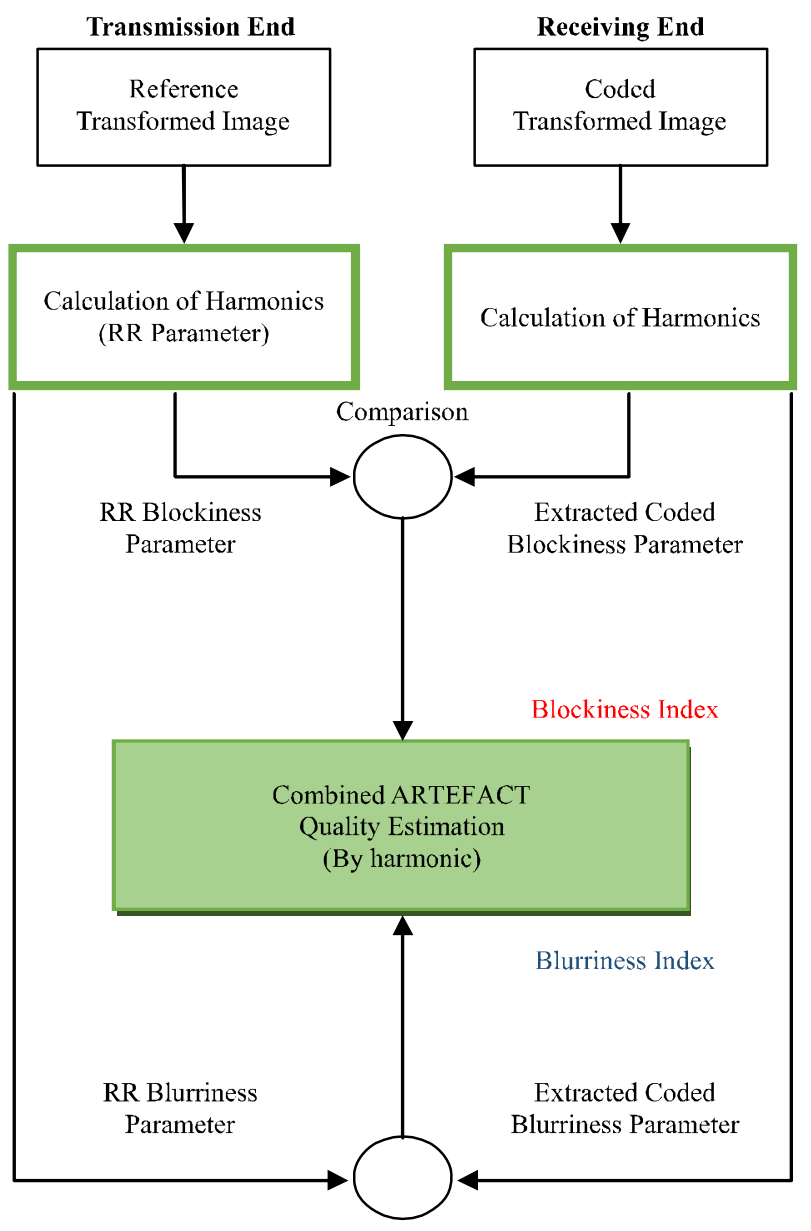

FIG. 4. SYSTEM MODEL OF IMAGE QUALITY ASSESSMENT INREDUCED REFERENCE MODE USING HARMONIC ANALYSIS by FFT, the other ac coefficients strength is calculated. The strength of other ac coefficients $\left(\mathrm{AC}_{\text {other_strength_Ref }}\right)$ between horizontal and vertical harmonics is computed as RR parameter at the transmission end. Also the computation for the strength of other ac coefficients at the receiving end, $\mathrm{AC}_{\text {other_strength_Cod }}$ is computed.Then reference image $\mathrm{RR}$ parameter, $\mathrm{AC}_{\text {other_strength_Ref }}$ is compared with the coded image $\mathrm{AC}_{\text {other_strength_cod }}$. For model 2 of Fig. 6 using other ac coefficients, for the case when $\mathrm{AC}_{\text {other_strength_Cod }}>\mathrm{AC}_{\text {other_strength_Ref' }}$,

Blockiness $=\left(\mathrm{AC}_{\text {other_strength_Cod }}-\mathrm{AC}_{\text {other_strength_Ref }}\right)$

and when $\mathrm{AC}_{\text {other_strength_Cod }}<\mathrm{AC}_{\text {other_strength_Ref }}$,

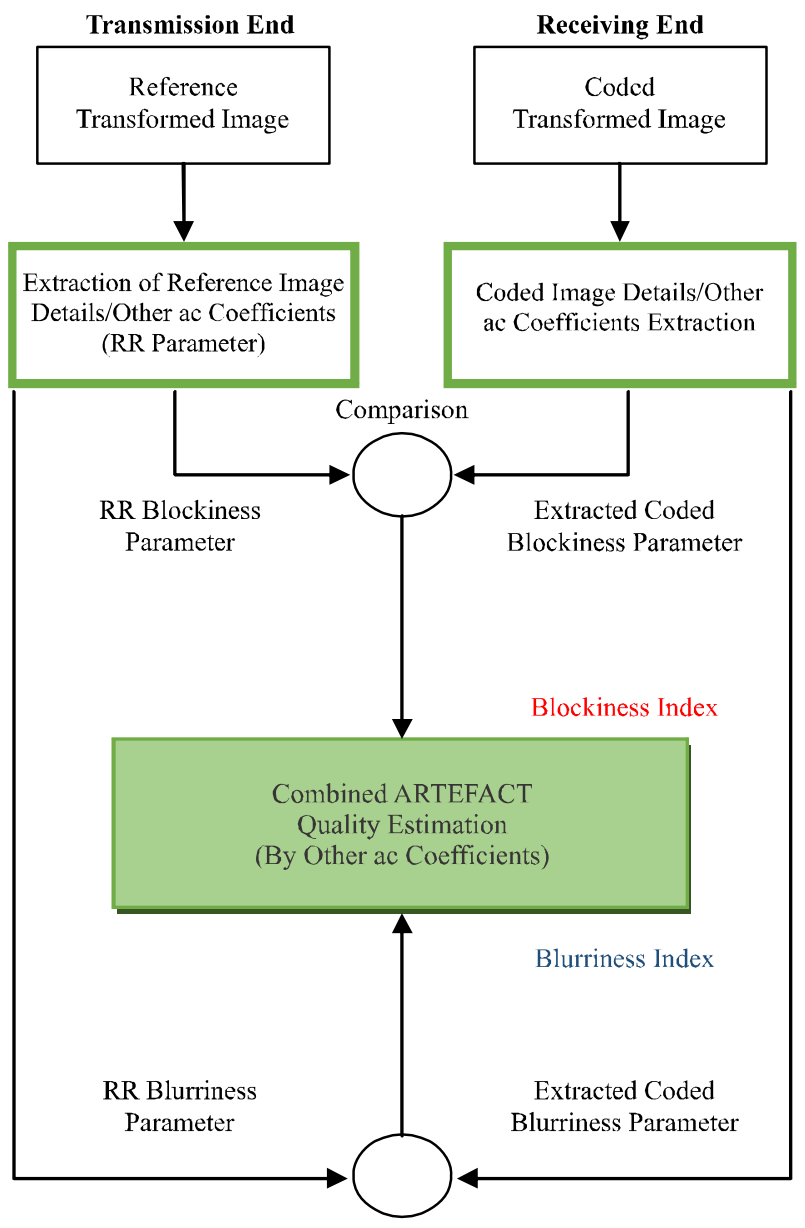

FIG. 5. SYSTEM MODEL OF IMAGE QUALITY ASSESSMENT INREDUCED REFERENCE MODE USING OTHER AC COEFFICIENTS

Mehran University Research Journal of Engineering \& Technology, Volume 36, No. 4, October, 2017 [p-ISSN: 0254-7821, e-ISSN: 2413-7219] 
Blurriness $=\mathrm{AC}_{\text {other_strength_Ref }}-\mathrm{AC}_{\text {other_strength_Cod }}$

The actual combined objective blockiness/blurriness artefacts value gives IQ metric, obtained by the summation of these two values using Equation (3).

\subsection{Image Quality Assessment using DC Coefficients with Other ac Coefficients in Frequency Domain}

System model of IQ assessment by combined blockiness and blurriness artefacts in RR mode using dc coefficients with other ac coefficients is shown in Fig. 7. In the image of 32x32 pixels blocks, other than harmonics and ac coefficients there is a dc coefficient block as well in the image. The strength of this dc coefficient is calculated and added with other ac coefficients of the image to measure more accurate results of blockiness and blurriness for IQ assessment.

For the model 3 of Fig. 7, when ACDC other_strength_Cod $^{>}$

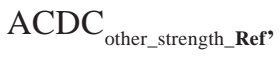

Blockiness $=\mathrm{ACDC}_{\text {other_strength_Cod }}-\mathrm{ACDC}_{\text {other_strength_Ref }}(8)$

and blurriness is obtained, when $\mathrm{ACDC}_{\text {other_strength_Cod }}<$

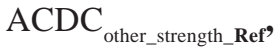

Blurriness $=\mathrm{ACDC}_{\text {other_strength_Ref }}-\mathrm{ACDC}_{\text {other_strength_Cod }}$

\section{Strength of Harmonics in Blocky Blocks}

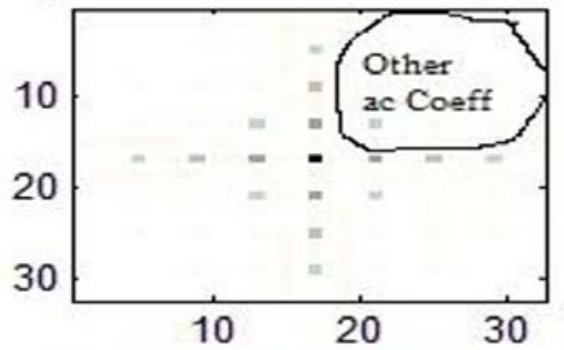

\section{$32 \times 32$ pixels image showing harmonics}

FIG. 6. FOURIER TRANSFORM OF IDEAL $32 X 32$ PIXELS BLOCKY IMAGE SHOWINGOTHER AC COEFFICIENTS
Final IQ metric of Equation (3) is obtained by the summation of these two objective blockiness/ blurriness artefacts values from Equations (8-9).

The above presented methods in 2.1, 2.2, 2.3 use the LIVE database2 [13] for 233 different coded image sequences. The database provides the DMOS (Differential Mean Opinion Scores) of each sequence. Then the test is performed for our designed algorithm of reduced reference DIQAM meter, objective blockiness values on the subjective DMOS scores of LIVE image database2. The method of blurriness measurement also uses the LIVE

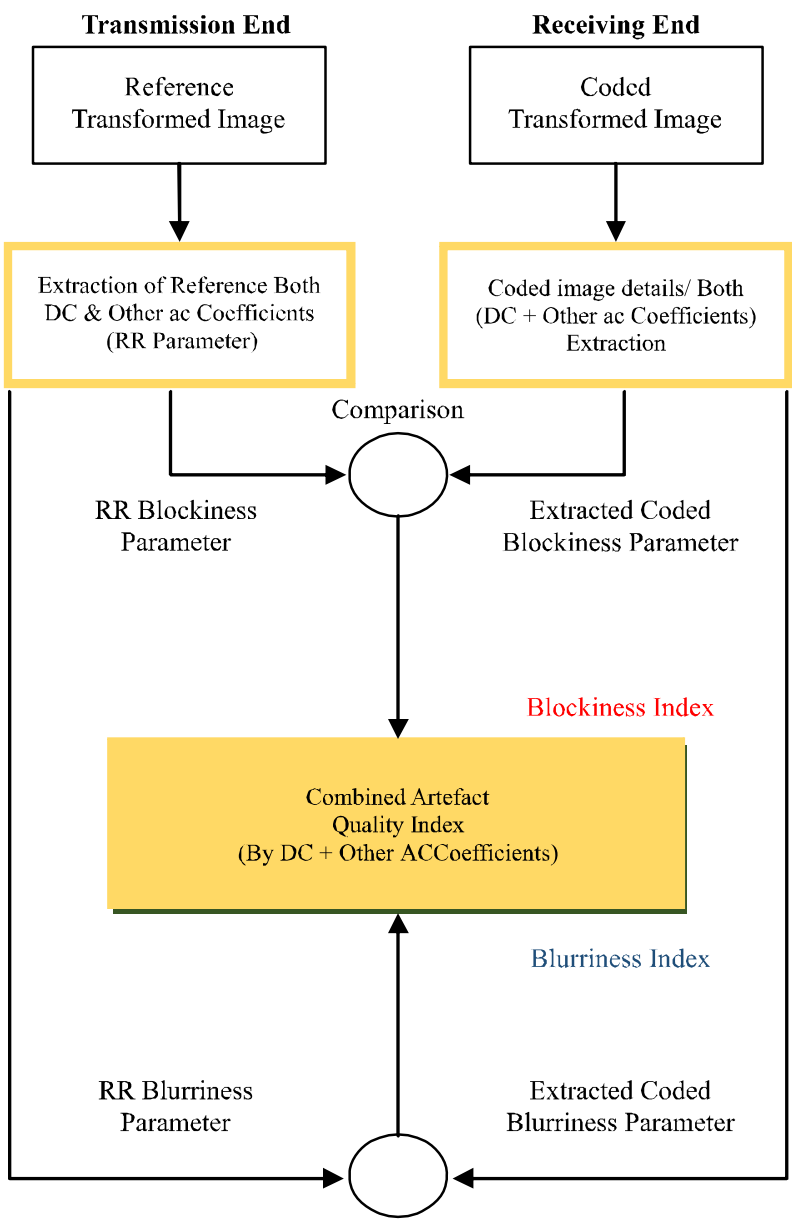

FIG. 7. SYSTEM MODEL OF IMAGE QUALITY ASSESSMENT IN REDUCED REFERENCE MODE USING DC COEFFICIENTS WITH OTHER AC COEFFICIENTS

Mehran University Research Journal of Engineering \& Technology, Volume 36, No. 4, October, 2017 [p-ISSN: 0254-7821, e-ISSN: 2413-7219] 
database2 [13] for 174 different blurred coded image sequences. The database has the blurriness values for each image. Ultimately, the testing has been done for our designed RR, objective blurriness values with the subjective blur scores of LIVE image database2.

\section{RESULTS}

The objective values of the blockiness and blurriness distortions for the designed IQ assessment meter are measured through the programming in Matlab software. The algorithm for RR blockiness and blurriness quality meter is developed. The designed DIQAM algorithm is tested on the LIVE image database2 [13]. By comparing the correlation coefficient of our blockiness and blurriness values with subjective DMOS scores, the quality of the designed algorithm for blockiness is proven.
Table 1 shows the DIQAM results of objective Blockiness correlation wih subjective DMOS scores for blocky LIVE database2. Column 3(blockiness due to both blocky and blurry blocks in image) shows, a matching of $94.69 \%$ is obtained using harmonic analysis and 91.76\%is obtained byusing other ac coefficients. But highest similarity $96.35 \%$ is achieved by using dc coefficients with other ac coefficients.

Similarly Table 2 represents the DIQAM results of objective Blurriness correlation wih subjective blur scores for blurry LIVE database2. It shows a matching of 93.67 and $91.65 \%$ by harmonic analysis and byusing other ac coefficients respectively. As for the case of blockiness, highest similarity of $93.50 \%$ for blurrinessdistortionis achieved by using dc coefficients with other ac coefficients.

TABLE 1. IMAGE QUALITY ASSESSMENT USING OBJECTIVE BLOCKINESS CORRELATION RESUTS WIH SUBJECTIVE DMOS SCORES FOR BLOCKY LIVE DATABASE2

\begin{tabular}{|c|c|c|}
\hline Image Details Required for Proposed DIQAM & $\begin{array}{c}\text { Blockiness } \\
\text { (Correlation Results wih LIVEDMOS Scores) } \\
\text { (Due to only Blocky Blocks in Image) } \\
(\%)\end{array}$ & $\begin{array}{c}\text { Blockiness } \\
\text { (Correlation Results wih LIVE DMOS Scores) } \\
\text { (Due to Both Blocky and Blurry Blocks in Image) } \\
\text { (\%) }\end{array}$ \\
\hline $\begin{array}{c}\text { For Model of Fig. 4 } \\
\text { By using Harmonics }\end{array}$ & 94.53 & 94.69 \\
\hline $\begin{array}{c}\text { For Model of Fig. 5 } \\
\text { By Average of(All Other AC/H+V) }\end{array}$ & 85.81 & 91.76 \\
\hline $\begin{array}{c}\text { For Model of Fig. 7 } \\
\text { By All Other AC/H+V + DC Coefficient }\end{array}$ & 92.31 & 96.35 \\
\hline
\end{tabular}

TABLE 2. IMAGE QUALITY ASSESSMENT USING OBJECTIVE BLURRINESS CORRELATION RESUTS WIH SUBJECTIVE BLUR SCORES FOR BLURRY LIVE DATABASE2

\begin{tabular}{|c|c|c|}
\hline Image Details required for Proposed DIQAM & $\begin{array}{c}\text { Blurriness } \\
\text { (Correlation Results wih LIVE Blur Scores) } \\
\text { (Due to only Blurry Blocks in Image) } \\
(\%)\end{array}$ & $\begin{array}{c}\text { Blurriness } \\
\text { (Correlation Results wih LIVE Blur Scores) } \\
\text { (Due to both Blocky andBlurryBlocks in Image) } \\
\text { (\%) }\end{array}$ \\
\hline $\begin{array}{c}\text { For Model of Fig. } 4 \\
\text { By using Harmonics }\end{array}$ & 93.60 & 93.67 \\
\hline $\begin{array}{c}\text { For Model of Fig. 5 } \\
\text { By Average of (All Other AC/H+V) }\end{array}$ & 89.74 & 91.65 \\
\hline $\begin{array}{c}\text { For Model of Fig. 7 } \\
\text { By All Other AC/H+V + DC Coefficient }\end{array}$ & 92.61 & 93.50 \\
\hline
\end{tabular}

Mehran University Research Journal of Engineering \& Technology, Volume 36, No. 4, October, 2017 [p-ISSN: 0254-7821, e-ISSN: 2413-7219] 


\section{CONCLUSION}

In this research work a novel low complexity RR, DIQAM method established on frequency domain transforms is proposed. The quality assessment method is easy to execute and efficiently estimates the blockiness and blurriness distortions in the images compressed using DCT (JPEG).The performance of the proposed IQ meter's objective blockiness and blurriness results is compared with LIVE image database2 subjective DMOS and blurred scores. The proposed metrics provide a comprehensive new objective IQ measurement method which also efficiently accommodates the available, image communication medium bandwidth. The obtained correlation results of around 94-96\% indicate that the proposed quality meter technique can be executed well in objective RRIQ assessment. It is appropriate technique for real-time applications.

\section{ACKNOWLEDGEMENT}

This reserach work was supported by the Sir Syed University of Engineering \& Technology, Karachi, Pakistan. The authors are thankful to the officials and colleagues for providing the expertise, laboratory and technical assistance during this research assignment. Authors also show our gratitude to the authorities who have given full cooperation during the course of completion of this research work.

\section{REFERENCES}

[1] ITU-RRecommendations BT. 500-11, "Methodology for the Subjective Assessment of the Quality of Television”, ITU-R, Geneva, Switzerland, 2002.

Wang, Z., and Bovik A.C., "Modern Image Quality Assessment”, Morgan \& Claypool Publishers, Volume 3, New York, December, 2006.

[3] Gastaldo, P., and Redi, J.A., "Machine Learning Solutions for Objective Visual Quality Assessment", 6 ${ }^{\text {th }}$ International Workshop on Video Processing and Quality Metrics for Consumer Electronics, 2012.
[4] Bhupinderjit, K., "Digital Imageand Video Compression Techniques”, International Journal of Emerging Technology and Advanced Engineering, Volume 3, No. 7, pp. 554-558, July, 2013.

[5] Wikipedia. Available: www.wikipedia.com, December 2015

[6] Ivanovici, M., Richard, N., and Fernandez-Maloigne, C., “Towards Video Quality Metrics Basedon Colour Fractal Geometry”, Journal on Image and Video Processing, Volume 4, 2010.

[7] Punchihewa, A., Bailey, D., and Hodgson, R., "Objective Quality Assessment of Coded Images: The Development of New Quality Metrics”, International Conference on Internet and Telecommunication, pp. 1-6, 2004.

[8] Eskicioglu, A.M., and Fisher P.S., "Image Quality Measures and their Performance”, IEEE Transactions on Communications, Volume 43, No. 12, pp. 2959-2965, December, 1995.

[9] Zhang, L., Zhang, L., Mou, X., and Zhang, D.,’FSIM: A Feature Similarity Index for Image Quality Assessment”, 2011.

[10] Damera-Venkata, N., Kite, T.D., Geisler, W.S., Evans, B.L., and Bovik A.C., "Image Quality Assessment Based on a Degradation Model”, Volume 9, No. 4, April, 2000.

[11] Sheikh, H.R., Sabir, M.F., and Bovik, A.C., “A Statistical Evaluationof Recent Full Reference Image Quality Assessment Algorithms", IEEE Transactions on Image Processing, 2006.

[12] Gao, X., Lu, W., Tao, D., and Li, X., "Image Quality Assessment Based on Multiscale Geometric Analysis”, IEEE Transactions on Image Processing, Volume 18, No. 7, pp. 1409-1423, July, 2009,

[13] LIVE Image Database. Available:http://live.ece.utexas.edu, December, 2015

[14] Haque, M.I., Qadri, M.T., and Siddiqui, N., "Reduced Reference Blockiness and Blurriness Meter for Image Quality Assessment”, The Imaging Science Journal, ManeyPublishing,Volume 63, No. 5, pp. 296-302, UK, June, 2015. 